13

\title{
Особенности взаимодействия молекул германа с поверхностью германия в вакууме и в присутствие потока водорода
}

\author{
(C) Н.Л. Ивина, ${ }^{1}$ К.А. Кондрашина ${ }^{2}$ \\ ${ }^{1} \mathrm{OOO}$ „MEPA HH“, \\ 603950 Нижний Новгород, Россия \\ ${ }^{2}$ Нижегородский государственный технический университет им. Р.Е. Алексеева, \\ 603950 Нижний Новгород, Россия \\ e-mail: nataivina@mail.ru
}

Поступило в Редакцию 23 ноября 2020 г.

В окончательной редакции 24 ноября 2020 г.

Принято к публикации 5 января 2021 г.

В диапазоне ростовых температур $(300-700)^{\circ} \mathrm{C}$ проведен анализ температурного поведения основных кинетических параметров, ответственных за скорости протекания на поверхности растущего слоя германия процессов пиролиза адсорбируемых молекул германа. Проведена оценка степени покрытия поверхности германия водородом и радикалами молекул германа. Установлена взаимосвязь характерной частоты пиролиза молекул гидридов со скоростью захвата молекул поверхностью и скоростью встраивания атомов Ge в растущий слой. Температурная зависимость скорости распада фрагментов молекул на поверхности германия обнаруживает немонотонное поведение, характер которого различен в разных температурных режимах и зависит от того, на каком этапе процесса сорбции происходит захват поверхностью водорода с адсорбируемой молекулы гидрида. Характеристики пиролиза германа сопоставляются с аналогичными характеристиками для пиролиза молекул силана. Обнаружено, что повышение концентрации рабочих газов в камере роста заметным образом сказывается на каталитических свойствах ростовой поверхности.

Ключевые слова: химическая эпитаксия, гидриды германия, поверхностный пиролиз, молекулярный и поверхностный водород, кинетические коэффициенты.

DOI: $10.21883 / J T F .2021 .06 .50875 .82-20$

\section{Введение}

Методы химической эпитаксии при пониженном давлении газов в реакторе в настоящее время активно применяются при выращивании полупроводниковых гетероструктур. Особое место в эпитаксиальной технологии занимают гетерокомпозиции на основе элементов 4-й группы, изготавливаемые с использованием водородсодержащих соединений. Их взаимодействие с эпитаксиальной поверхностью обусловливает специфику протекающих на поверхности роста физико-химических процессов, отвечающих за скорость наращивания слоев. Отклонение ростовых характеристик от стандартного поведения, описываемого простейшими моделями, требует проведения детального анализа всей совокупности явлений, протекающих как в объеме реактора, так и на поверхности растущей пленки.

Кинетика поверхностных процессов, включающая процессы сорбции разнообразных фрагментов молекул, взаимодействующих с ростовой поверхностью рабочих газов, на сегодняшний день более-менее полно изучена только для поверхности пленки эпитаксиального кремния, выращиваемой с использованием потоков силана и дисилана [1-3]. Для гидридов кремния в широком температурном интервале определены кинетические константы, ответственные за скорости протекания на поверхности роста основных физико-химических процес- сов [4-6]. В связи с проявляемым интересом к проблеме гетероэпитаксии на кремнии достаточно толстых (сотни нанометров) слоев чистого $\mathrm{Ge}$ и сплавов $\mathrm{Si}_{1-x} \mathrm{Ge}_{x}$ c повышенным содержанием германия [7] в последние годы наметился интерес к изучению особенностей поверхностного пиролиза молекул гидридов германия как на поверхности $\mathrm{Si}[8]$, так и на поверхности растущего слоя германия [9-11]. В то же время несмотря на схожесть молекул гидридов кремния и германия, для гидридов германия по ряду аспектов кинетики их поверхностного пиролиза и влияния на ростовой процесс окончательной ясности еще не достигнуто. Это затрудняет не только понимание закономерностей, проявляющихся на температурных зависимостях скорости роста пленок $\mathrm{Ge}$, выращиваемых разными методами с использованием гидридов германия [12,13], но и понимание ряда особенностей, отличающих рост из гидридов слоев $\mathrm{Ge}$ от роста слоев $\mathrm{Si}$ [14]. Для прогнозирования ростовых характеристик и понимания особенностей, наблюдаемых на них, требуется знание кинетических коэффициентов, отвечающих за скорости физикохимических процессов, протекающих на поверхности. В литературе, однако, информация даже по основным кинетическим коэффициентам, отвечающих за захват и поверхностный распад молекул германа и необходимая для объяснения наблюдаемых закономерностей, практически отсутствует. 
Ниже нами на базе представленной на сегодняшний день в литературе экспериментальной информации по выращиванию пленок германия из моногермана методом высоковакуумной (UHVCVD) газофазной эпитаксии проведен анализ особенностей ростовых характеристик, отличающих при гомоэпитаксии низкотемпературный рост пленок германия из моногермана от роста пленок кремния с использованием моносилана. Для сопоставления приведены также данные по использованию в эпитаксиальном процессе более привлекательных для производства проточных методов, использующих в реакторе пониженное по сравнению с атмосферным давление газа носителя. С целью последующего выявления механизмов особенностей, наблюдаемых на технологических кривых, на базе имеющихся экспериментальных данных в интервале рабочих температур проведена оценка кинетических констант, отвечающих за скорости протекания на ростовой поверхности основных физико-химических процессов. Для анализа использован метод $[6,15,16]$, в основе которого лежат модельные расчеты, выполняемые на базе системы кинетических уравнений, принимающей во внимание адсорбируемые ростовой поверхностью молекулярные фракции распадающихся молекул.

\section{1. Особенности гомоэпитаксии слоев германия с использованием моногермана}

При разработке кинетических моделей с целью выявления наиболее важных физико-химических процессов, протекающих на эпитаксиальной поверхности в ходе проведения ростовых экспериментов, наиболее часто апеллируют к измеряемым зависимостям скорости роста слоя от разнообразных технологических параметров (температура подложки, давления газов, характеристики поверхности и другие). Поэтому, прежде всего, представляется важным рассмотреть имеющиеся в литературе данные по скоростям роста слоев германия при их выращивании в вакууме из гидридов германия при пониженной температуре. Ниже на рис. 1 по аналогии с кривыми, приведенными в работе [22] для роста слоев кремния из моносилана, представлены полученные в различных технологических группах зависимости скорости роста слоев германия от температуры (рис. 1, $a$ ) и от давления германа в реакторе (рис. 1,b). Кривые на рис. 1 построены для UHVCVD-метода (газофазная технология в сверхвысоком вакууме $\left(P_{\mathrm{GeH}_{4}} \leq 0.1 \mathrm{~Pa}\right)$ на основе данных работ $[10,13,11,18,19]$, для LPCVD (газофазная технология при пониженном $\left(P_{\mathrm{H}_{2}}<10^{2} \mathrm{~Pa}\right)$ давлении смеси гидрида с водородом) на основе данных работы [21] и RPCVD (газофазная проточная технология при уменьшенном $\left(10^{2}<P_{\mathrm{H}_{2}}<10^{4} \mathrm{~Pa}\right)$ давлении водорода в реакторе) на основе данных работ $[7,9,12,17,20]$. При построении температурных зависимостей экспериментальные данные (символы на рис. 1,a), аппроксими-
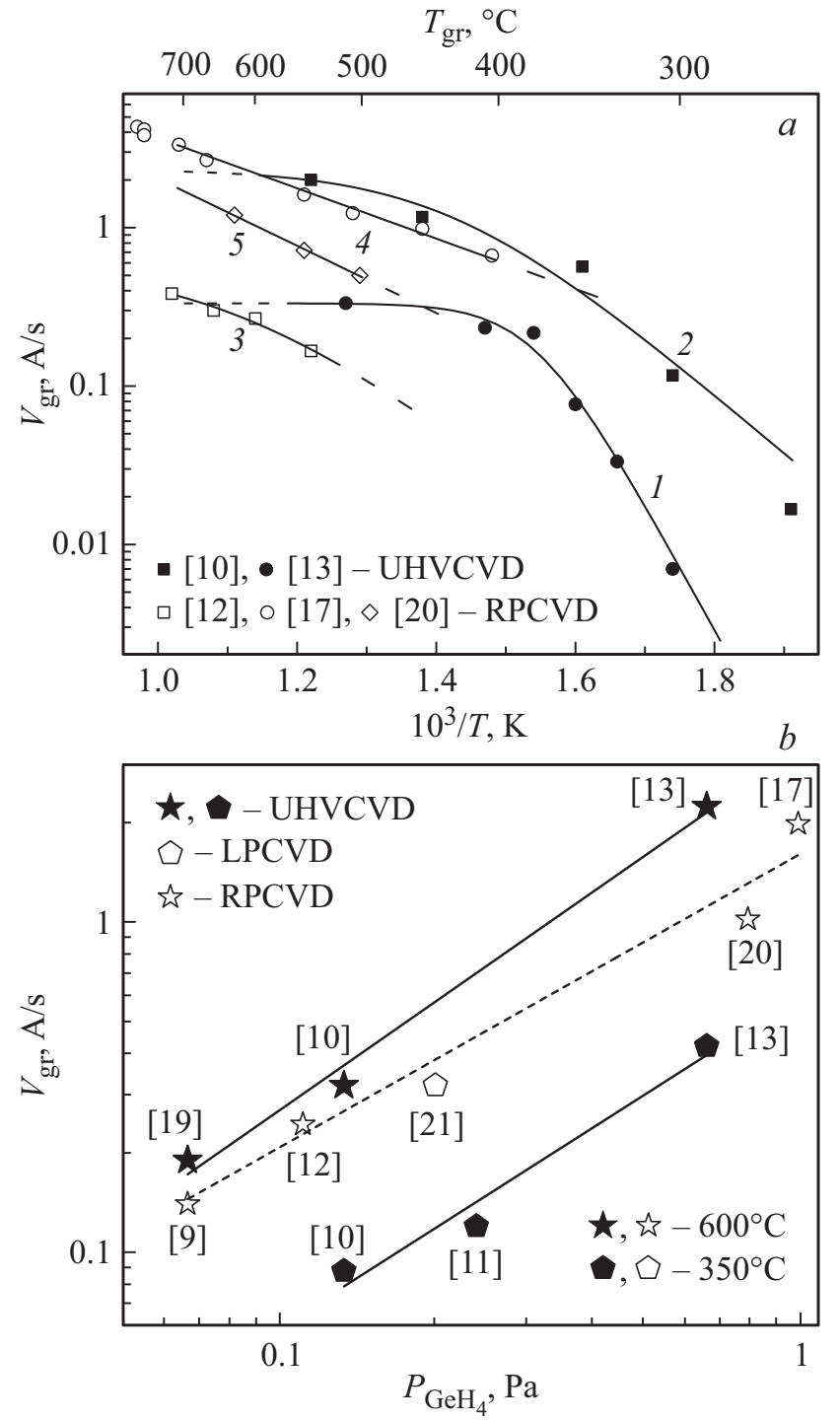

Рис. 1. Зависимости скорости роста слоев германия (a) от температуры при заданном давлении газов, равных: UHVCVD $-P_{\mathrm{GeH}_{4}}(\mathrm{~Pa})=0.133 \quad(\mathbf{\square}[10]-$ кривая 1), $0.666(\bullet[13] \quad-$ кривая 2), RPCVD $P_{\mathrm{GeH}_{4}}(\mathrm{~Pa}) / P_{\mathrm{H}_{2}}(\mathrm{~Pa})=0.11 / 2666(\square[12]-$ кривая 3), 1/2666 ( [17] — кривая 4), 0.8/800 ( $\diamond$ [20] — кривая 5); символы данные, взятые из соответствующих работ, кривые 1-5 аппроксимации соотношением (1) экспериментальных точек; и зависимости скорости роста слоев германия $(b)$ от парциального давления германа в реакторе при заданной температуре роста $T_{g r}$ равной $600^{\circ}$ и $350^{\circ} \mathrm{C}$, символы - данные, соответствующие конкретным значениям давлений газов в реакторах установок UHVCVD и L(R)PCVD и температуре роста, взятые из работ: $P_{\mathrm{GeH}_{4}}(\mathrm{~Pa}) / P_{\mathrm{H}_{2}}(\mathrm{~Pa})=0.133 / 0$ [10], 0.666/0 [13], 0.24/0 [11], 0.0666/0 [19] - UHVCVD; 0.2/10 [21] - LPCVD; $0.0666 / 1333$ [9], 0.11/2666 [12], 0.8/800 [20], 1/2666 [17] RPCVD; линии на рисунке - линейные аппроксимации экспериментальных точек.

ровались (кривые на рис. $1, a)$ функцией

$$
\left.V_{\mathrm{gr}}(\mathrm{A} / \mathrm{s})=V_{0} /\left\{1+\exp \left[\left(T_{0}-T_{g r}\right) / \Delta T\right)\right]\right\}
$$


со значениями параметров $V_{0}(\mathrm{~A} / \mathrm{s}), T_{0}\left({ }^{\circ} \mathrm{C}\right), \Delta T\left({ }^{\circ} \mathrm{C}\right)$ соответственно равными: $0.319,371,21.7$ [10]; 2.49, 455.3, 66.2 [13]; 0.5, 604.1, 90.6 [12]; 45.2, 1199.5, 194,5 [17].

На рис. $1, b$ представлены зависимости скорости роста слоев германия от давления германа в реакторе в UHVCVD-методе роста и от парциального давления германа в реакторе RP(LP)CVD-установок. Выбор осей на рис. $1, b$ связан с удобством сопоставления соответствующих кривых для роста пленок германия и пленок кремния [22]. B CVD-установках проточного типа связь между стандартно измеряемым на входе системы расходом газов и соответствующим давлением газов в реакторе, согласно [23], описывается соотношением $P_{\mathrm{GeH}_{4}}(\mathrm{~Pa})=10^{3}\left(F_{\mathrm{GeH}_{4}} / F_{\mathrm{H}_{2}}\right) \times P_{\text {tot }}(\mathrm{Pa})$, где $F_{\mathrm{GeH}_{4}}(\mathrm{sccm})$ и $F_{\mathrm{H}_{2}}(\mathrm{slm})$ расход германа и водорода в газовых линиях, а $P_{\text {tot }} \approx P_{\mathrm{H}_{2}}-$ полное давление газа в ректоре во время роста.

Аналогично росту пленок кремния из моносилана [22] при выращивании пленок германия L(R)PCVD-методом (рис. $1, b)$ при заданной температуре эпитаксии скорость роста пленки Ge растет с увеличением парциального давления прекурсора $\left(\mathrm{GeH}_{4}\right)$, оставаясь, однако, несколько ниже скорости роста германия UHVCVD-методом. Наблюдаемый для RPCVD-метода при $T_{g r} \geq 600^{\circ} \mathrm{C}$ незначительный разброс экспериментальных точек, полученных для разных значений давлений $\left(P_{\mathrm{H}_{2}}\right)$ газа носителя (от 800 [20] до $2666 \mathrm{~Pa}$ [24]), относительно линейной зависимости $V_{g r}\left(P_{\mathrm{GeH}_{4}}\right)$ - штриховая кривая на рис. $1, b$ указывает на слабую зависимость скорости роста слоя германия от давления молекулярного водорода в реакторе установки при повышенных температурах. При низких температурах эпитаксии (порядка $350^{\circ} \mathrm{C}$ ) влияние поверхностного водорода на скорость роста проявляется более заметно. Для низких давлений молекулярного водорода (в частности, при разбавлении прекурсора $\mathrm{GeH}_{4}$ водородом) наблюдается увеличение скорости роста пленки Ge (cp. данные работ [11] и [21]), вследствие активного участия водорода в процессе поверхностного пиролиза гидрида [6]. Однако дальнейшее повышение давления газа носителя в реакторе установки и термический распад водорода на ростовой поверхности приводят к заполнению свободных поверхностных связей атомарным водородом, обусловливая резкое падение скорости роста пленки при низких температурах роста [24].

В большинстве экспериментов по выращиванию из моногермана при пониженном давлении в диапазоне от 500 до $700^{\circ} \mathrm{C}$ слоев германия с ростом температуры в отличие от эпитаксии слоев кремния не наблюдалось насыщения скорости роста пленки Ge. Выход на участок насыщения скорости роста с повышением температуры, характерный для выращивания пленок кремния из моносилана [6,14], при использовании моногермана имел место лишь при высоком (близком к атмосферному) давлении водорода в CVD-реакторе [24,25], а в UHVCVD-установках при использовании в качестве рабочего газа дигермана [26]. Все это указывает на то, что, несмотря на схожесть молекул моногермана и моносилана, полная идентичность процесса их поверхностного пиролиза на соответствующих поверхностях отсутствует. Для процессов распада молекул германа на поверхности растущего слоя германия это может приводить не только к низкотемпературному сдвигу изучаемых зависимостей и понижению характерных величин активационных параметров, но и к изменению роли механизмов, отвечающих за протекание поверхностных реакций и традиционных для пиролиза гидридов $\mathrm{Si}$ на поверхности кремния.

\section{2. Десорбция водорода с поверхности германия}

В области пониженных ростовых температур ключевым моментом при исследовании физико-химических процессов на поверхности роста слоя является анализ свойств и характеристик водородных комплексов, адсорбируемых ростовой поверхностью и оказывающих доминирующее влияние на протекающие на поверхности реакции. Поэтому одними из важнейших характеристик, наиболее часто обсуждаемыми в литературе и в значительной степени определяющими поведение скорости роста слоя в области низких температур, являются степень покрытия поверхности водородом и соответствующий ей коэффициент десорбции водорода.

Нужно отметить, что на сегодняшний день в литературе отсутствуют данные по определению степени покрытия $\theta_{\mathrm{H}}\left(T_{g r}\right)$ ростовой поверхности германия водородом в условиях проводимых технологических экспериментов, подобные тем, что были получены для покрытия водородом ростовой поверхности $\mathrm{Si}$ [14]. И это несмотря на то, что на сегодняшний день надежно установлено, что адсорбируемый поверхностью Ge слоя водород при пониженных температурах эпитаксии играет весьма важную роль, изменяя каталитические свойства поверхности и улучшая ее морфологию [10]. В отличие от ситуации с кремнием [27] сегодня в литературе имеется всего лишь несколько публикаций по прямому определению методом термоуправляемой десорбции коэффициента десорбции водорода $\chi_{\mathrm{H} / \mathrm{Ge}}$ с поверхности Ge [28-30]. Полученные в указанных работах результаты позволяют все же сделать вывод, что зависимость $\theta_{\mathrm{H}}\left(T_{g r}\right)$ на поверхности $\mathrm{Ge}$, аналогично $\mathrm{Si}$, с достаточной точностью может быть описана соотношением:

$$
\theta_{\mathrm{H}}\left(T_{g r}\right)=1 /\left\{1+\exp \left[\left(T_{g r}-T_{0}^{\prime}\right) / \delta T\right]\right\} .
$$

Для поверхностей $\mathrm{Si}$ и $\mathrm{Ge}$ основное отличие проявляется в сдвиге (примерно на $200^{\circ} \mathrm{C}$ [28]) температурной зависимости $\theta_{\mathrm{H}}\left(T_{g r}\right)$ на поверхности германии в область более низких температур (рис. 2,a). В формуле (2) величина сдвига связана с параметром $T_{0}^{\prime}$. Значения параметров $T_{0}^{\prime}$ и $\delta T$ в формуле (2) нетрудно найти путем сопоставления выражения для $\chi_{\mathrm{H} / \mathrm{Ge}}$, получаемого 

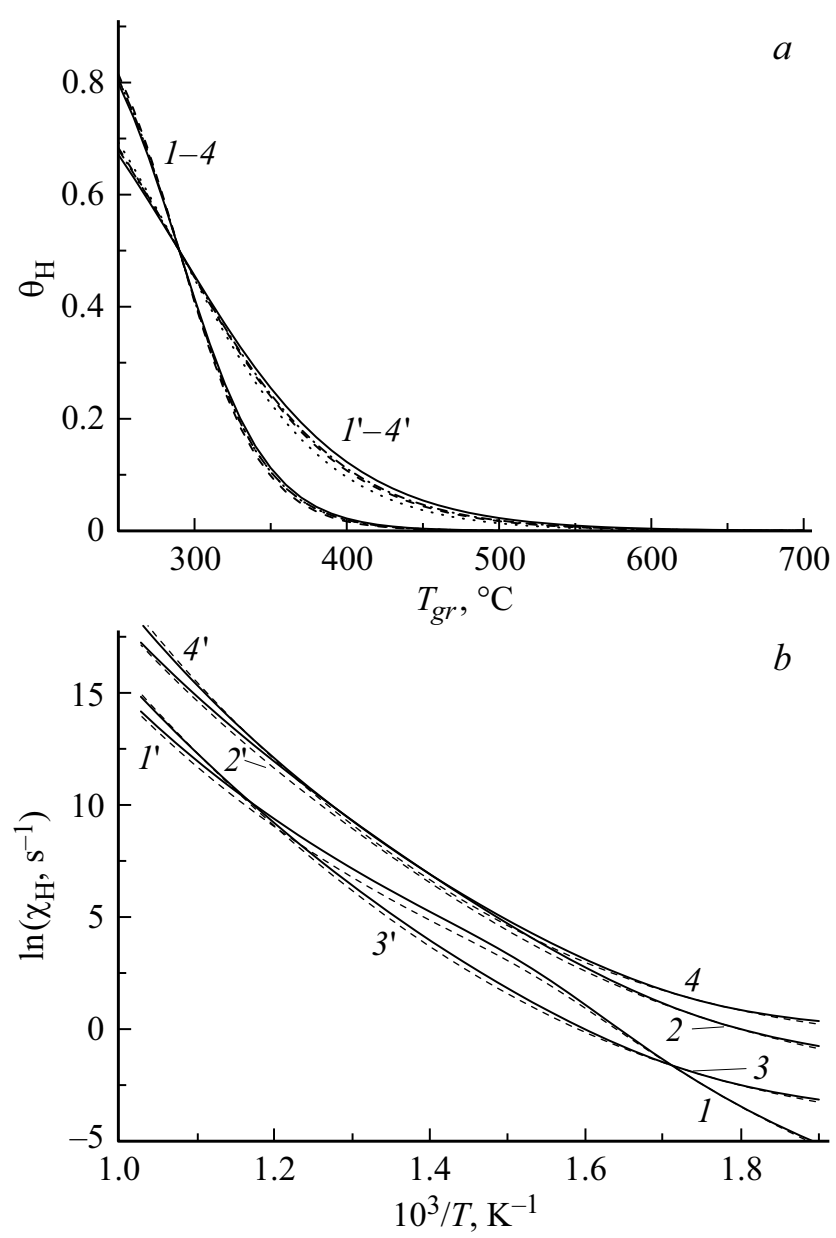

Рис. 2. Зависимости степени покрытия поверхности германия водородом от температуры $(a)$, рассчитанные по формуле (3) с параметрами $\left(T_{0}^{\prime}, \Delta T\right)$, равными $T_{0}^{\prime}=290, m=1(1-4)$, $\delta T=1-29,2-27,3,4-28 ; m=2\left(1^{\prime}-4^{\prime}\right), \delta T=1^{\prime}-$ $56,2^{\prime}-52,3^{\prime}-53,4^{\prime}-49 ; 1,1^{\prime}-$ сплошные линии [10], $2,2^{\prime}$ - штриховые линии [13], 3, 3' - штрих-пунктирные линии [12], 4, 4' - пунктирные линии [17], и соответствующие им зависимости коэффициента десорбции $\chi_{\text {н }}$ от обратной температуры $(b)$ для $m=1-$ сплошные линии $(1-4)$ и $m=2-$ штриховые линии $\left(1^{\prime}-4^{\prime}\right)$ со значениями $\operatorname{Ln} \chi_{0}$, лежащими в интервале $35-38$, и энергией активации $E_{\mathrm{H}}^{a} \approx 1.7-1.74 \mathrm{eV}$.

из кинетических уравнений [6,27]:

$$
\chi_{\mathrm{H} / \mathrm{Ge}}=1 /\left(l_{0} m !\right)(k+n) V_{g r} / \theta_{\mathrm{H}}^{m},
$$

c активационной зависимостью стандартного вида $\chi_{\mathrm{H} / \mathrm{Ge}}=\chi_{0} \exp \left(-E_{\mathrm{H}}^{a} / k_{B} T_{g r}\right)$. Здесь $E_{\mathrm{H}}^{a}-$ энергия активации процесса десорбции водорода с поверхности германия. Ее значение $E_{\mathrm{H}}^{a}=1.735 \mathrm{eV}(40 \mathrm{kkal} / \mathrm{mol})$ и значение предэкспоненциального множителя $\chi_{0}=4 \cdot 10^{13} \mathrm{~s}^{-1}$ получены в работе [29] методом термодесорбционной спектрометрии. В формуле (3) величина $l_{0}$ равна отношению объемной $\left(n_{0}\right)$ и поверхностной $\left(n_{s}\right)$ концентраций атомов $\mathrm{Ge}$ в пленке $\left(l_{0}=n_{0} / n_{s}\right), m$ коэффициент, характеризующий форму (ассоциативная $m=1$, либо диссоциативная $m=2$ ) десорбции водо- рода с поверхности, $k(n)$ - число атомов водорода, переходящих с молекулы германа $\mathrm{GeH}_{4}$ на поверхность германия на предхемосорбционной (хемосорбционной) стадиях пиролиза [6]. Зависимости $\operatorname{Ln} \chi_{\mathrm{H} / \mathrm{Ge}}$ от обратной температуры, рассчитанные с использованием формулы (3) со значениями параметров $k=1, n=3, m=1$ (сплошные линии), 2 (штриховые линии), и соответствующие им зависимости $\theta_{\mathrm{H}}\left(T_{g r}\right)$ для представленных на рис. 1,a кривых $V_{g r}\left(T_{g r}\right)$ приведены на рис. 2. Расчеты показывают, что изменение параметров $k$ и $n$ приводит лишь к изменению величины $\chi_{0}$, не меняя значения энергий активации процесса десорбции. С высокой степенью уверенности можно предположить, что при малом содержании водорода на поверхности роста слоя Ge, например, при использовании UHVCVD-метода, более вероятной является десорбция водорода в атомарной форме $(m=1)$. При повышенном содержании водорода в объеме реактора и на поверхности роста, в частности, при использовании LPCVD-метода, более вероятной является десорбция водорода в молекулярной форме $(m=2)$. Окончательный ответ, однако, может быть получен только путем экспериментального определения степени покрытия поверхности $\mathrm{Ge}$ водородом (см., например, [14]) и сопоставления экспериментальных данных с теорией (кривые на рис. 2,a). Ниже для контроля полученных значений параметров нами были использованы также дополнительные критерии, основанные на результатах более полного кинетического анализа.

\section{3. Коэффициент захвата молекул германа поверхностью слоя германия}

Другим наиболее часто обсуждаемым в литературе кинетическим коэффициентом, лимитирующим величину скорости роста слоя в области повышенных температур, является коэффициент захвата молекул газа поверхностью роста пленки. Прямое определение коэффициента захвата ростовой поверхностью молекул рабочего газа является весьма трудоемким и дорогостоящим [31]. Весьма ненадежными являются и расчеты коэффициента адсорбции с использованием квантово-химических уравнений [1-4], где обычно применяются сильно упрощенные модели, далекие от реалий проводимых технологических экспериментов по выращиванию слоев. Результаты расчетов зависят от параметров приповерхностного потенциала и определяются не только типом фрагмента адсорбируемой молекулы и способом его посадки на поверхностные атомы, но и от расположения атомов в глубинных приповерхностных слоях кристалла. Более простым способом оценки коэффициента захвата молекул газа ростовой поверхностью, традиционно применяемым в литературе, является использование выражений, получаемых на базе простейшей системы кинетических 
уравнений. При этом в подавляющем большинстве случаев ограничиваются балансом между адсорбционной по гидриду и десорбционной по водороду составляющими [5,30,32,33]. Предполагается, что первый механизм отвечает за рост пленки при высокой температуре. Второй механизм, связанный с присутствием на ростовой поверхности водорода, определяет характер роста пленки при пониженных температурах роста. При этом слагаемыми в кинетических уравнениях, содержащими адсорбируемые поверхностью фрагменты молекулы гидрида, обычно пренебрегают, что предполагает полный распад адсорбируемых молекул на отдельные атомы уже на первой стадии процесса пиролиза. Согласно этой модели, выражение для коэффициента захвата может быть представлено в виде (см, например, [6]):

$$
\mathrm{S}_{\mathrm{GeH}_{4}}=V_{g r}\left(T_{g r}\right) / l_{0}\left(k_{0}+1\right) ! F_{\mathrm{GeH}_{4}}\left(1-\theta_{\mathrm{H}}\right)^{k_{0}+1} .
$$

Здесь $F_{\mathrm{GeH}_{4}}\left(\mathrm{~cm}^{-2} \mathrm{c}^{-1}\right)$ - поток молекул германа к поверхности роста пленки, $k_{0}-$ число атомов водорода, переходящих с молекулы гидрида на поверхность. В условиях молекулярного течения газов поток молекул $F_{\mathrm{GeH}_{4}}$ связан с давлением гидрида $P_{\mathrm{GeH}_{4}}(\mathrm{~Pa})$ в реакторе вакуумной установки соотношением $F_{\mathrm{GeH}_{4}}=4.68 \cdot 10^{21} \cdot P_{\mathrm{GeH}_{4}} /\left(M_{\mathrm{GeH}_{4}} T_{\mathrm{GeH}_{4}}\right)^{1 / 2}$, где $T_{\mathrm{GeH}_{4}}\left({ }^{\circ} \mathrm{C}\right)$ - температура газа в реакторе, $M_{\mathrm{GeH}_{4}}-$ молекулярный вес германа. Для потока молекул германа имеем: $F_{\mathrm{GeH}_{4}}=37.2 P_{\mathrm{GeH}_{4}}$. В области повышенных температур покрытие поверхности водородом пренебрежимо мало $\left(\theta_{\mathrm{H}} \rightarrow 0\right)$ и коэффициент захвата непосредственно связан с измеряемыми на практике величинами: давлением газа в реакторе и скоростью роста слоя. В области пониженных температур также требуется знание степени покрытия поверхности адсорбируемыми атомами водорода. Используя соотношение (4) и результаты, представленные в разд. 1, 2, проведем оценку коэффициента захвата $S_{\mathrm{GeH}_{4}}$ в диапазоне ростовых температур. Соответствующие результаты для условий технологических экспериментов, приведенных на рис. 1, представлены на рис. $3, a$, где $T(\mathrm{~K})=T_{g r}+273^{\circ}$ С. Изменение давления германа для обоих методов роста, как следует из рис.3, b, слабо сказывается на величине коэффициента захвата молекул германа поверхностью.

Представленные на рис. 3 результаты позволяют провести оценку абсолютной величины коэффициента захвата $S_{\mathrm{GeH}_{4}}$ молекул германа поверхностью Ge пленки в разных диапазонах ростовых температур и для разных методов роста. В литературе в соответствие с простейшей кинетической моделью пиролиза (4) коэффициент захвата молекул гидрида ростовой поверхностью в области повышенных температур эпитаксии, соответствующих условию $\theta_{\mathrm{H}} \approx 0$, обычно предполагается слабо зависящим от температуры, что обусловливает наблюдаемое постоянство скорости роста слоя при повышенных температурах эпитаксии [10,26]. Соответственно из вида кривых 1,2 на рис. 3 следует, что только в методе UHVCVD в диапазоне повышенных
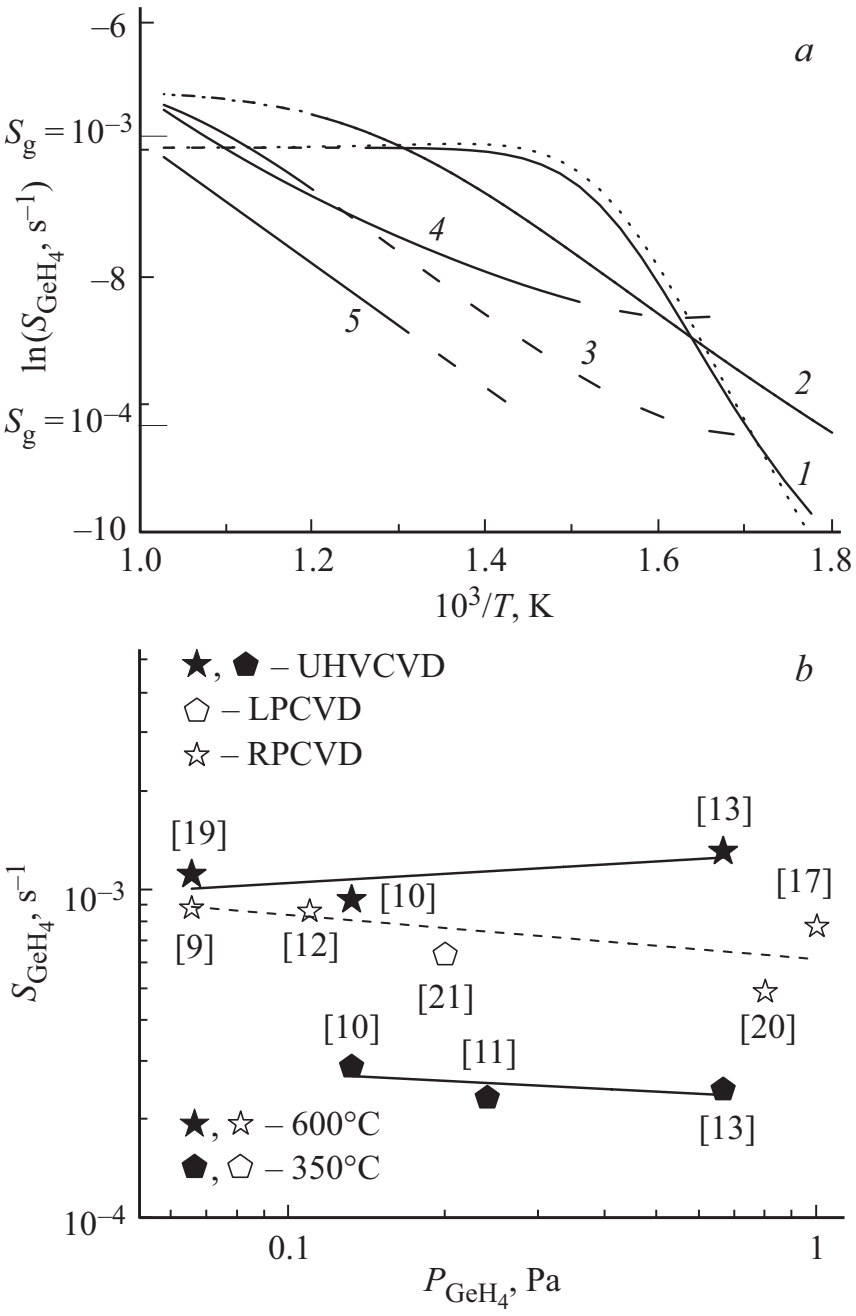

Рис. 3. Температурные зависимости (a) скорости поверхностного распада молекул германа $\mathrm{GeH}_{4}$, рассчитанные по формуле (4) для $k_{0}=0, m=1$ - сплошные (штриховые аппроксимация на более широкий температурный диапазон) линии, $m=2-$ пунктирные линии, с использованием данных, представленных на рис. $1, a$. Кривые $1-5-$ соответствуют кривым $1-5$ на рис. $1, a ; b-$ зависимости скорости захвата ростовой поверхностью молекул германа от парциального давления германа в реакторе установок при заданной температуре роста $T_{g r}$, равной 600 и $350^{\circ} \mathrm{C}$, фигуры - соответствуют символам, использованным на рис. $1, b$; линии - линейные аппроксимации экспериментальных точек.

температур и предельно низких давлений коэффициент захвата молекул германа поверхностью германия остается практически постоянным и равным величине $0.001-0.002 \mathrm{~s}^{-1}$, что близко к значению коэффициента захвата молекул силана поверхностью кремния. Постоянство скорости роста слоя Ge при повышенных температурах эпитаксии $\left(T_{g r}>450^{\circ} \mathrm{C}\right)$, обусловливающее слабую зависимость от температуры коэффициента адсорбции, наблюдается, однако, только для низких давлений газа в UHVCVD-реакторе (кривые 1 на рис. 1, a и 3). Температурная зависимость коэффици- 
ента захвата молекул гидрида $\mathrm{Ge}(\mathrm{Si})$ и соответственно скорости роста слоя $\mathrm{Ge}(\mathrm{Si})$, обычно связываемая с покрытием поверхности адсорбируемым водородом (4), проявляется лишь при пониженных температурах роста (для германия при $T_{g r}$ ниже $450^{\circ} \mathrm{C}$ ). В то же время в большинстве экспериментов с использованием RPCVD-реактора по выращиванию слоев Ge из германа монотонный рост функции $V_{g r}\left(T_{g r}\right)$ (кривые 2-5 на рис. $1, a)$ с повышением температуры наблюдается во всем диапазоне ростовых температур, в том числе и при температурах выше $500^{\circ} \mathrm{C}$. Основываясь на кривых, представленных на рис. 3, можно провести оценку энергии активации процесса захвата молекул германа в области $T_{g r}>450^{\circ} \mathrm{C}$, где вид зависимости $S_{\mathrm{GeH}_{4}}\left(T_{g r}\right)$ близок к линейному. Аппроксимируя температурную зависимость $S_{\mathrm{GeH}_{4}}$ стандартным образом: $S_{\mathrm{GeH}_{4}}=S_{0} \exp \left(-E_{a}^{S} / k_{B} T\right)$, и полагая ассоциативную форму десорбцию водорода $(m=1)$ для $E_{a}^{S}(\mathrm{eV})$, получаем значения: $E_{a}^{S}(\mathrm{eV})=0.0038$ (1), 0.17 (2), 0.36 (3), $0.28(4), 0.39(5)$. С ростом давления газов в реакторе энергия активации адсорбционного процесса (соответствующая высоте барьера в приповерхностном потенциале) повышается от $0.004 \mathrm{eV}$ для предельно низкого давления германа в UHVCVD-методе (кривая 1) до $0.3-0.4 \mathrm{eV}$ для проточого RPCVD-метода (кривые 3-5). Видно, что присутствие водорода в смеси газов в рамках простейшей кинетической модели оказывает заметное влияние на адсорбционный потенциал поверхности, обусловливая его более высокие значения. Нужно отметить, что и квантово-химические расчеты, выполненные, в частности, для моносилана [32-35], даже в условиях сверхвысокого вакуума показывают сильную зависимость конечного результата от вида поверхностного потенциала, обусловливая значительный разброс значений высоты активационного барьера от 0.14 [32] до 0.72 [33], 0.6 [34], $0.8 \mathrm{eV}$ [35] при использовании разного вида функционала плотности. Таким образом, можно ожидать, что присутствие в потоке (смеси) газа водорода будет оказывать заметное влияние на каталитические свойства поверхности и соответственно на величины энергий активаций процессов захвата и распада молекул гидрида.

В принципе появление температурной зависимости как скорости роста, так и коэффициента захвата может быть обусловлено и другими причинами, например, зависимостью потока молекул, поступающих к ростовой поверхности, от температуры. В частности, более низкая по сравнению с моносиланом энергия распада молекул моногермана может приводить вследствие взаимодействия молекул газа с нагретыми элементами реактора к появлению в потоке молекул газа к поверхности дополнительного потока атомов германия. Тогда общий поток рабочих молекул к ростовой поверхности в (4) можно представить в виде $S_{\mathrm{GeH}_{4}} F_{\mathrm{GeH}_{4}} \rightarrow S_{\mathrm{GeH}_{4}} F_{\mathrm{GeH}_{4}}+$ $+S_{\mathrm{Ge}} F_{\mathrm{Ge}}$, где коэффициенты захвата $S_{g}$ не зависят от температуры. Для величины $S_{\mathrm{GeH}_{4}}$, согласно рис. 3, ориентировочно имеем $S_{\mathrm{GeH}_{4}} \sim 0.001$, для величины
$S_{\mathrm{Ge}}$ в соответствии с общепринятыми представлениями, используемыми в кинетических моделях при описании процессов в вакуумных реакторах с атомарными пучками, будем полагать $S_{\mathrm{Ge}} \sim 1$. С ростом температуры подложки отношение атомарного потока к молекулярному будет расти, обусловливая наблюдаемый рост с температурой характеристик системы в области повышенных ростовых температур. Составляющую атомарного потока в зависимости от температуры можно оценить, исходя из разности скоростей роста слоя $\mathrm{Ge}$ при низкой $\left(T_{g r}^{\min } \sim\right.$ $\left.450^{\circ} \mathrm{C}\right)$ и высокой температурах $T_{g r}\left(T_{g r}^{\min } \sim 400^{\circ} \mathrm{C}<T_{g r}\right.$ $<T_{g r}^{\max } \sim 700^{\circ} \mathrm{C}$ ) роста слоя. Отношение скоростей роста соответственно равно: $V_{g r}\left(T_{g r}\right)=V_{g r}\left(T_{g r}^{\min }\right)=1+$ $+S_{\mathrm{Ge}} F_{\mathrm{Ge}}\left(T_{g r}^{\max }\right) / S_{\mathrm{GeH}_{4}} F_{\mathrm{GeH}_{4}}\left(T_{g r}\right)$. Отсюда для потока $F_{\mathrm{Ge}}\left(T_{g r}\right)$ получаем $F_{\mathrm{Ge}}\left(T_{g r}\right)=\left\{V_{g r}\left(T_{g r}\right) / V_{g r}\left(t_{g r}^{\min }\right)-1\right\} /$ $\left[S_{\mathrm{GeH}_{4}} F_{\mathrm{GeH}_{4}}\left(T_{g r}\right) / S_{\mathrm{Ge}}\right]$.

$\mathrm{C}$ ростом температуры доля атомов $\mathrm{Ge}$ в общем молекулярном потоке к поверхности увеличивается от минимальной величины до значения, определяемого температурой горячих поверхностей. В диапазоне ростовых температур от 400 до $700^{\circ} \mathrm{C}$ скорость роста слоя германия в RPCVD-методе, согласно рис. 1, $a$, возрастает примерно на порядок. Отсюда следует, что максимальная (при $T_{g r}=T_{g r}^{\max } \sim 700^{\circ} \mathrm{C}$ ) величина потока атомов $\mathrm{Ge}$ к поверхности подложки составляет величину порядка $1 \%$ от полного потока молекул германа.

\section{4. Особенности накопления на поверхности фрагментов молекул германа}

Согласно проводимым расчетам по взаимодействую гидридов кремния и германия с поверхностью $\mathrm{SiGe}$ слоя, физико-химические процессы, протекающие на поверхности германия, во многом идентичны тем, что наблюдаются на поверхности кремния при взаимодействии последней с молекулярным пучком гидрида кремния. Они включают в себя захват поверхностью газообразной молекулы и последующий ее распад с образованием на поверхности различных фрагментов молекулы гидрида и атомарного водорода. На следующей стадии происходит распад адсорбированных радикалов молекулы. Параллельно важную роль играют процессы десорбции поверхностного водорода и процессы встраивания адатомов $\mathrm{Ge}$ в растущий слой. В соответствие со сказанным общая схема физико-химических реакций, протекающих на ростовой поверхности и определяющая процесс пиролиза молекул, участвующих в ростовом процессе, является более сложной, чем предполагалось при оценке коэффициента захвата молекулы поверхностью. Для однокомпонентной модели пиролиза, принимающей во внимание присутствие на ростовой поверхности только одного долгоживущего фрагмента $\mathrm{GeH}_{j}(j=1-3)$ мо- 
лекулы германа, она может быть представлена в виде

$$
\begin{gathered}
\mathrm{GeH}_{4}(g)+\underline{k+1} \rightarrow \underline{\mathrm{GeH}_{j}}+\underline{k \mathrm{H}}+(2-(j+k) / 2) \mathrm{H}_{2} \uparrow, \\
\underline{\mathrm{GeH}_{j}}+\underline{n} \rightarrow \underline{\mathrm{Ge}}+\underline{n \mathrm{H}}+((j-n) / 2) \mathrm{H}_{2} \uparrow, \\
\underline{n \mathrm{H}} \rightarrow(n / 2) \mathrm{H}_{2} \uparrow ; \quad \underline{\mathrm{Ge}} \rightarrow \mathrm{Ge}(\mathrm{cr}) .
\end{gathered}
$$

Здесь параметр $k(0 \leq k \leq 4-j)$ - учитывает число атомов водорода с молекулы, захватываемых поверхностью на предхемосорбционной стадии процесса пиролиза, параметр $n(0 \leq n \leq j)$ - число атомов водорода, переходящих на поверхность с адсорбируемого фрагмента молекулы гидрида, подчеркивание указывает на наличие связи атома (молекулы) с поверхностью. Ниже мы обсудим только наиболее обсуждаемую схему поверхностного пиролиза германа с наиболее стабильным фрагментом $\mathrm{GeH}_{3}$ (гермил).

Соответствующую выше приведенной схеме пиролиза (5) систему кинетических уравнений для безразмерных поверхностных концентраций $\theta_{x x}$ фрагментов распада молекул гидрида, отнесенных к полному числу адсорбционных центров $n_{s}$ на чистой поверхности растущего слоя $\mathrm{Ge}$, по аналогии с работами $[6,15]$, представим в следующем виде:

$$
\begin{gathered}
\partial \theta_{\mathrm{GeH}_{j}} / \partial t=(k+1) !\left(S_{\mathrm{GeH}_{4}} F_{\mathrm{GeH}_{4}} / n_{s}\right)\left(\theta_{n b l}\right)^{k+1} \\
-(n+1) ! v_{\mathrm{GeH}_{j}} \theta_{\mathrm{GeH}_{j}}\left(\theta_{f r}\right)^{n}, \\
\partial \theta_{\mathrm{H}} / \partial t=k(k+1) !\left(S_{\mathrm{GeH}_{4}} F_{\mathrm{GeH}_{4}} / n_{s}\right)\left(\theta_{n b l}\right)^{k+1} \\
\quad+n(n+1) ! v_{\mathrm{GeH}_{j}} \theta_{\mathrm{GeH}_{j}}\left(\theta_{f r}\right)^{n}-m ! \chi_{\mathrm{H}} \theta_{\mathrm{H}}^{m}, \\
\partial \theta_{\mathrm{Ge}} / \partial t=(n+1) ! v_{\mathrm{GeH}_{j}} \theta_{\mathrm{GeH}_{j}}\left(\theta_{f r}\right)^{n}-r_{\mathrm{Ge}} \theta_{\mathrm{Ge}}, \\
\theta_{\mathrm{GeH}_{j}}+\theta_{\mathrm{Ge}}+\theta_{\mathrm{H}}+\theta_{f r}=1, \quad \theta_{f r}=\theta_{b l}+\theta_{n b l} .
\end{gathered}
$$

Здесь $\theta_{\mathrm{GeH}_{j}}$ соответствуют безразмерным концентрациям адсорбируемых поверхностью радикалов гидрида германия, $\theta_{\mathrm{Ge}}-$ концентрации адатомов германия на поверхности, $\theta_{\mathrm{H}}-$ концентрации поверхностного водорода, $\theta_{f r}$ - концентрации свободных поверхностных связей. Последние включают в себя связи, как блокированные $\left(\theta_{b l}=j \theta_{\mathrm{GeH}_{j}}\right)$, так и неблокированные $\left(\theta_{n b l}=\theta_{f r}-\theta_{b l}\right)$ радикалами молекулы германа, захваченных поверхностью. Факториальные множители в (6) соответствуют числу перестановок, т.е. числу возможных конфигураций мест двумерной поверхностной решетки, на которые можно разместить адсорбируемые молекулы и атомы. Как и выше, индекс $m$ равен единице, если атомы водорода десорбируют с поверхности слоя поодиночке, и 2, если в момент десорбции происходит их связывание в молекулу водорода. Кинетические константы $S_{\mathrm{GeH}_{4}}, v_{\mathrm{GeH}_{j}}, \chi_{\mathrm{H}}, r_{\mathrm{Ge}}$, входящие в систему уравнений (2) и имеющие размерность частоты $\left(\mathrm{s}^{-1}\right)$, отвечают за скорости захвата $\left(S_{\mathrm{GeH}_{4}}\right)$ и распада $\left(v_{\mathrm{GeH}_{j}}\right)$, захваченного поверхностью радикала молекулы $\mathrm{GeH}_{j}$, десорбцию водорода $\left(\chi_{\mathrm{H}}\right)$ и встраивание $\left(r_{\mathrm{Ge}}\right)$ адатомов $\mathrm{Ge}$ в растущий слой. Запись уравнений кинетики в представленном виде позволяет провести учет как типа поверхностной кристаллической решетки, так и зависимости кинетических коэффициентов от способа перевода атомов водорода с молекулы гидрида на поверхностные состояния. Поверхностная концентрация адатомов германия связана через коэффициент встраивания $r_{\mathrm{Ge}}$ со скоростью роста пленки $V_{g r}$ соотношением: $V_{g r}(\AA / \mathrm{s})=l_{0} r_{\mathrm{Ge}} \theta_{\mathrm{Ge}}$. Для $l_{0}$ ниже принято значение: $l_{0}=\left(n_{s} / n_{0}\right) \cdot 10^{-8} \approx 0.12 \mathrm{~nm}$. В соответствии с используемой методикой анализа наиболее явные отличия в поведении искомых кинетических коэффициентов связаны с различиями в характере температурного поведения скорости роста пленок, а также с особенностями заполнения поверхностных связей адсорбируемыми атомами водорода. При известных кинетических константах система уравнений (6) позволяет рассчитать поверхностные концентрации продуктов распада молекул для различных схем процесса пиролиза.

Наиболее просто поверхностные концентрации фрагментов распадающейся молекул находятся для стационарного случая, если решения уравнений (6) выразить через следующие независимые параметры:

$$
\begin{gathered}
\alpha=V_{g r} / l_{0}, \quad \beta=\theta_{n b l}^{(k+1)}=\alpha /\left((k+1) ! S_{\mathrm{GeH}_{4}} F_{\mathrm{GeH}_{4}} / n_{s}\right), \\
\gamma=\left(1-\beta^{1 /(k+1)}-\theta_{\mathrm{H}}\right) .
\end{gathered}
$$

Последние, в свою очередь, определяются через значения задаваемых технологических параметров $F_{\mathrm{GeH}_{4}}$ и $T_{g r}$ и измеряемые в процессе роста слоя характеристики процесса, такие как скорость эпитаксиального наращивания слоя германия $V_{g r}$ и степень покрытия поверхности $\mathrm{Ge}$ водородом $\theta_{\mathrm{H}}$. Использование в уравнениях кинетики (6) величин $\alpha, \beta, \gamma$ в качестве независимых параметров позволяет легко получить соотношения для расчета поверхностных концентраций $\theta_{x x}$ :

$$
\begin{gathered}
\theta_{\mathrm{Ge}}=\alpha / r_{\mathrm{Ge}}, \quad \theta_{\mathrm{GeH}_{j}}=\left(\gamma-\theta_{\mathrm{Ge}}\right) /(j+1), \\
\theta_{f r}=1-\theta_{\mathrm{GeH}_{j}}-\theta_{\mathrm{Ge}}-\theta_{\mathrm{H}} .
\end{gathered}
$$

Характерный вид температурной зависимости концентрации радикалов $\theta_{\mathrm{GeH}_{3}}$, адсорбируемых ростовой поверхностью $\mathrm{Ge}$, для условий экспериментов, использованных на рис. $1, a$, и значений $S_{\mathrm{GeH}_{4}}$, представленных на рис. 3, показан на рис. 4. Нужно отметить, что в силу того, что значения $\theta_{\mathrm{GeH}_{3}}$ лежат в диапазоне от 0 до 1 , рассматриваемая зависимость $\theta_{\mathrm{GeH}_{3}}\left(T_{g r}\right)$ может быть использована в качестве дополнительного критерия истинности выбранных в разд. 2 и 3 параметров. В частности, использование бо́льших значений параметра $T_{0}$ в формуле (2) приводит к сдвигу кривых $\theta_{\mathrm{GeH}_{3}}$ на рис. 4 в сторону более высоких температур, отсекая тем самым диапазон низких температур вследствие появления нефизических решений $\left(\theta_{\mathrm{GeH}_{3}}<0\right)$. Расширяет область отрицательных значений $\theta_{\mathrm{GeH}_{3}}$ и использование при расчетах более низких значений коэффициента захвата $S_{\mathrm{GeH}_{4}}$, получаемого, например, из формулы (4) 


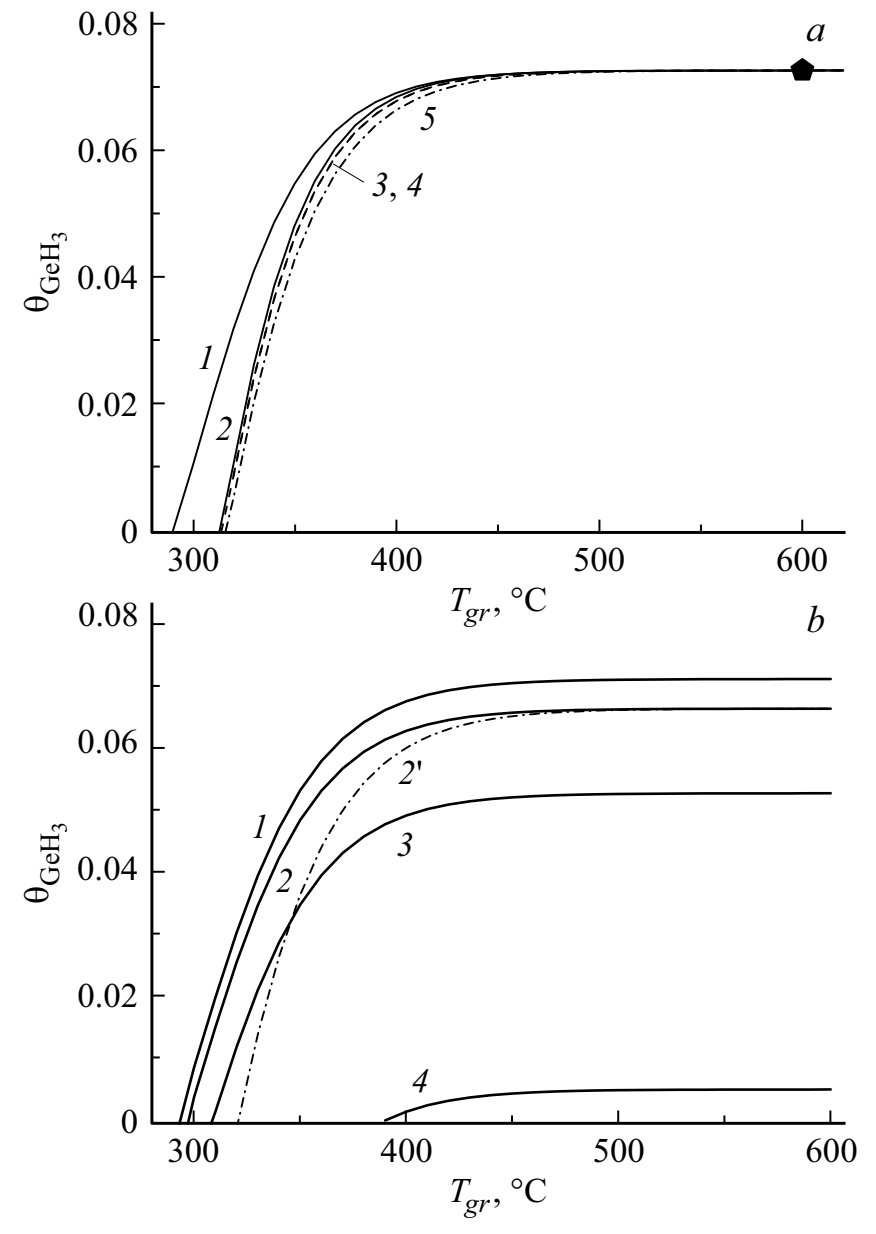

Рис. 4. Температурные зависимости поверхностных концентраций радикала $\mathrm{GeH}_{3}$, адсорбируемого поверхностью германия, рассчитанные в рамках кинетической модели $(\mathrm{jkn})$ $=(313), \mathrm{m}=1: a$ - для условий, соответствующих кривым 1-5 на рис. $1, a$, и значении коэффициента встраивания $r_{0}=300$, номера кривых совпадают с номерами кривых на рис. $1, a ; b$ - для условий работы [10] - сплошные линии и работы [20] - штрих-пунктирная линия при значениях параметра $r_{0}$, равного $r_{0}=100$ (кривая 1 ), 30 (кривые 2,2'), 10 (кривая 3), 3 (кривая 4). Символ на рис. $a-$ результат расчета, на основе данных, представленных в работах $[18,19]$.

при значении параметра $k_{0} \geq 1$. К ограничению области положительных значений $\theta_{\mathrm{GeH}_{3}}$, как показано на рис. $4, b$, будут приводить и сильно заниженные значения параметра $r_{0}$, отвечающего за скорость встраивания адатомов Ge в растущий слой. При уменьшении параметра $r_{0}$ концентрация гермила резко понижается в диапазоне высоких ростовых температур. Реально допустимые значения параметра $r_{0}$, отвечающего за слоевой механизм роста слоя, обычно соответствуют его высоким значениям, т. е. $r_{0} \gg 1$, однако меньших соответствующих значений для коэффициента встраивания атомов $\mathrm{Si}$.

Выше при расчетах поверхностной концентрации молекул гермила были использованы значения коэффициентов захвата, определяемые формулой (4) (рис. 3) и зависящие от температуры. При кинетическом анализе в области высоких температур и низких давлений германа коэффициент захвата молекулы германа чистой поверхностью германия можно предположить постоянным и близким к значениям коэффициента захвата молекулы силана поверхностью кремния, т.е. $S_{\mathrm{GeH}_{4}} \approx 0.001-0.002 \mathrm{~s}^{-1}$. Приемлемые значения поверхностной концентрации гермила (на уровне $\theta_{\mathrm{GeH}_{3}} \approx 0.07$ при $\left.T_{g r}>500^{\circ} \mathrm{C}\right)$ получаются при выборе усредненных (независящих от температуры) значений параметра $S_{\mathrm{GeH}_{4}}=0.001 \mathrm{~s}^{-1}$ для условий работы $[10], 0.0015 \mathrm{~s}^{-1}$ для условий работы [13], $\sim 0.001 \mathrm{~s}^{-1}$ для условий роста, соответствующих работе [17]. Таким образом, значения $S_{\mathrm{GeH}_{4}} \approx 0.001-0.002 \mathrm{~s}^{-1}$ наиболее приемлемы для условий, реализуемых в высоковакуумных установках. Для условий, реализуемых в ростовых системах проточного типа, коэффициент $S_{\mathrm{GeH}_{4}}$ характеризуется сильной зависимостью от температуры, что, как указывалось выше, может быть связано с влиянием молекулярного водорода на каталитические свойства поверхности.

\section{5. Скорость поверхностного распада адсорбируемых молекул гермила}

Весьма важным для прогнозирования нестационарного ростового процесса, особенно при использовании смеси газов, является параметр $v_{\mathrm{GeH}_{j}}$, ответственный за скорость распада на поверхности эпитаксиальной пленки адсорбируемых фрагментов молекул (в рассматриваемом случае молекул гермила $\mathrm{GeH}_{3}$ ). Вследствие сложности экспериментального определения данного параметра (современные методы позволяют измерять только концентрации адсорбируемого поверхностью радикала молекул гидрида) в литературе соответствующая информация на сегодняшний день отсутствует. Однако, как показано нами ранее на примере кинетики пиролиза молекул гидридов кремния на поверхности кремния $[6,15,16]$, провести оценку указанных величин и получить информацию о характере их температурного поведения можно более простым методом, проведя кинетический анализ результатов выполненных технологических экспериментов. Используя уравнения (6) и вытекающие из них соотношения (7),(8), нетрудно установить связь скорости распада $v_{\mathrm{GeH}_{j}}$ радикалов молекул германа на поверхности пленки Ge c коэффициентом встраивания $r_{\mathrm{Ge}}$ и перечисленными выше (8) параметрами системы. Соответствующее выражение имеет вид

$$
\begin{aligned}
& v_{\mathrm{GeH}_{j}}=\left\{j(1+1 / j)^{n+1} /(n+1) !\right\} \alpha\left\{\left(\gamma-\alpha / r_{\mathrm{Ge}}\right)\right. \\
& \left.\times\left[\gamma+(1+1 / j) \beta^{1 /(k+1)}-\alpha / r_{\mathrm{Ge}}\right]^{n}\right\} .
\end{aligned}
$$

Применение для анализа более сложных многокомпонентных кинетических моделей значительно усложняет задачу, делая, из-за недостаточного объема информации по выращиванию из гидридов планарных слоев 

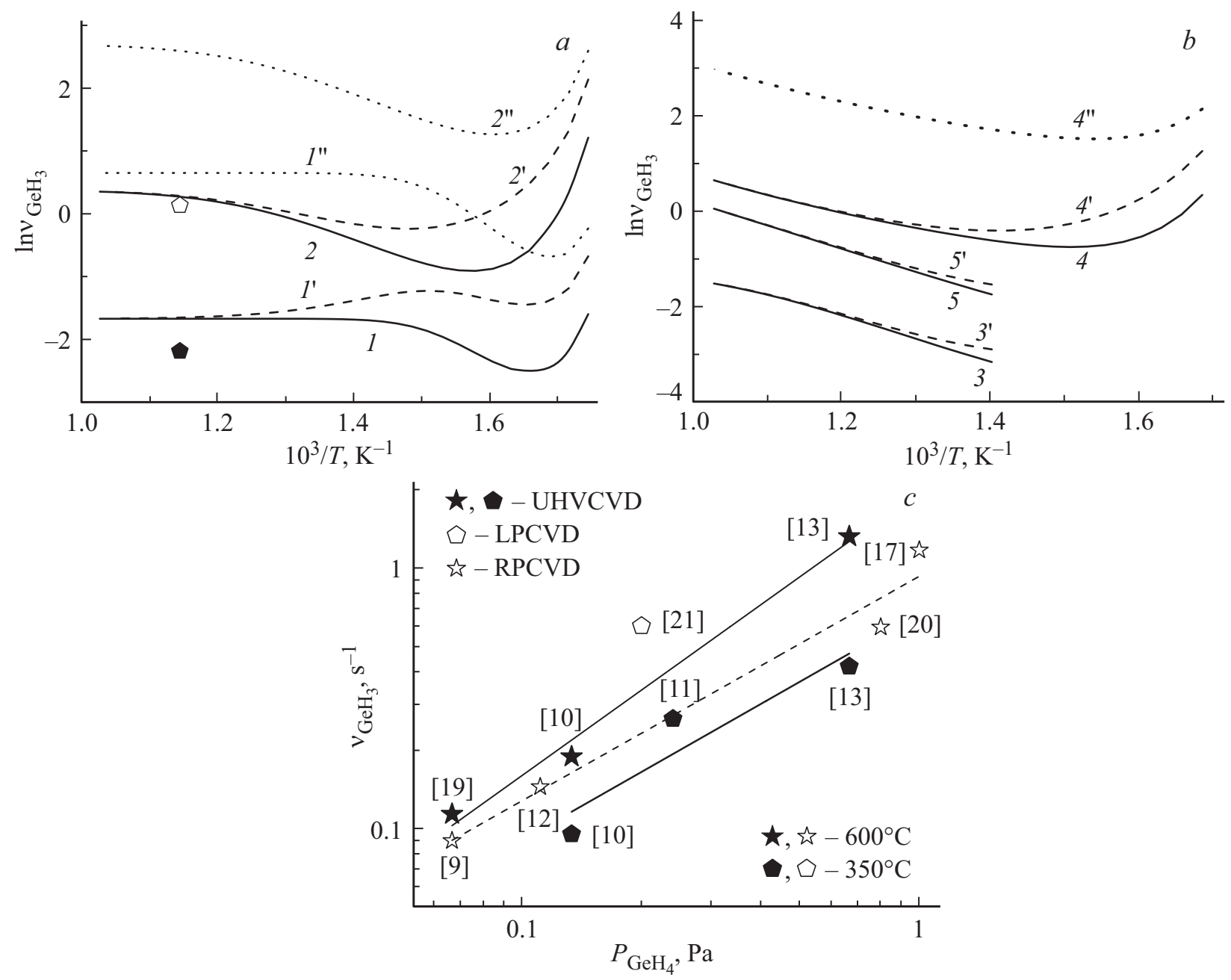

Рис. 5. Температурные зависимости скорости поверхностного распада молекул гермила $\mathrm{GeH}_{3}$, полученные в рамках кинетической модели с параметрами: $j=3, k=1, n=3, m=1-$ сплошные кривые $(1-5), m=2$ - штриховые кривые $\left(1^{\prime}-5^{\prime}\right) ; m=1$, $n=1$ - пунктирные кривые $\left(1^{\prime \prime}, 2^{\prime \prime}, 4^{\prime \prime}\right)$, для условий экспериментов, использованных в работах: $a[10,13]-$ соответствуют кривым $(1,2)$ на рис. $1, a ; b-[12,17,20]-$ соответствуют кривым $(3-5)$ на рис. $1, a$. Для других параметров выбраны значения: $a, b-T_{0}^{\prime}=290, \delta T-$ соответствуют кривым, представленным на рис. 2 ; параметр $S_{\mathrm{GeH}_{3}}$ выбран в соответствие с кривыми на рис. 3. Символы $(a)$ - результаты расчета на основе данных работ $[18,19]$ для $m=1$ (пятиугольник заштрихованный), $m=2$ (пятиугольник незаполненный); $c$ - зависимости скорости распада адсорбируемых молекул гермила от парциального давления германа в реакторе установок при заданной температуре роста $T_{g r}$ равной 600 и $350^{\circ} \mathrm{C}$, фигуры - соответствуют символам, использованным на рис. $1, b$ и рис. $3, b$; линии - линейные аппроксимации экспериментальных точек.

германия, использование таких моделей для расчетов преждевременными.

В применяемом на практике интервале ростовых температур для рассматриваемых кинетических параметров трудно ожидать выполнение закона Аррениуса с одной энергией активации. Причиной этого являются разные условия протекания поверхностных физико- химических процессов в области низких и высоких температур. Разным температурным интервалам соответствуют разные механизмы взаимодействия молекул с поверхностью и разные пути и способы распада фрагментов молекул на атомарно чистой (при $T_{g r}>500^{\circ} \mathrm{C}$ ) и частично заполненной водородом (при $T_{g r}<400^{\circ} \mathrm{C}$ ) поверхности пленки. Проводимые расчеты позволяют, прежде всего, для разных температурных интервалов провести оценку характерных времен распада накапливаемых на ростовой поверхности фрагментов молекул рабочего газа. Они также на качественном уровне позволяют судить о возможных путях и механизмах взаимодействия адсорбируемых молекул с ростовой поверхностью, и влияния на них различных факторов. Используя формулу (9), проанализируем вид температурной зависимости скорости распада молекул гермила для стандартных условий ростовых экспериментов, представленных в разд. 2 на рис. 1. Характерный вид соответствующих кривых, рассчитанных в интервале ростовых температур $300-700^{\circ} \mathrm{C}$ для модели пиролиза с поверхностным радикалом $\mathrm{GeH}_{3}$, представлен на рис. 5. Сопоставление кри- 
вых с номерами $i=(1-5)$ на рис. $5, a, b$ с соответствующими кривыми $i^{\prime}=\left(1^{\prime}-5^{\prime}\right)$, прежде всего, показывает, что изменение с температурой характера десорбции с поверхности водорода, т. е. выбор параметра $m$, равным 1 или 2 , сказывается на качественном виде зависимости $v_{\mathrm{GeH}_{j}}\left(T_{g r}\right)$ только в области пониженных ростовых температур, приводя к небольшому расхождению кривых с номерами $i$ и $i^{\prime}$. Слабое влияние на качественный вид кривых $v_{\mathrm{GeH}_{j}}\left(T_{g r}\right)$ оказывает и характер температурного поведения параметра $S_{\mathrm{GeH}_{4}}\left(T_{g r}\right)$. Изменение числа атомов водорода $(n)$, переводимых с захваченной молекулы на поверхность роста, при заданных величинах скорости роста, концентрации адсорбируемого на поверхности водорода и других параметров модели не меняет качественный вид температурной зависимости величины $v_{\mathrm{GeH}_{j}}$ во всем интервале температур. Однако уменьшение параметра $n$ повышает скорость распада адсорбируемых молекул в несколько раз (примерно на порядок величины при замене (рис. 5, $a, b$ ), например, параметра $n=3$ (сплошные кривые $1,2,4$ на $n=1$ (штриховые кривые $1^{\prime \prime}, 2^{\prime \prime}, 4^{\prime \prime}$ ).

Проведенные нами расчеты на рис. 5, $a, b$ выполнены, вообще говоря, для принципиально разных условий роста, соответствующих разным давлениям германа и молекулярного водорода в реакторе UHVCVD- и RPCVDустановок. Присутствие молекулярного водорода в ростовой камере даже при неизменном давлении германа и фиксированной температуре роста оказывает заметное влияние на ростовой процесс. В общем случае, присутствие повышенного количества водорода в реакторе установки может оказывать влияние не только на температурное поведение коэффициента захвата молекул гидрида, но и явиться причиной проявления одновременно нескольких механизмов последующей десорбции атомарного водорода с ростовой поверхности.

Кривые на рис. $5, a, b$ демонстрируют явную зависимость скорости поверхностного распада адсорбируемых молекул гермила от температуры роста. Наиболее близким к традиционному поведению, характерному для высокотемпературного роста пленок кремния, является эпитаксиальный рост слоев германия в условиях высокого вакуума при повышенных $\left(T_{g r}>500^{\circ} \mathrm{C}\right)$ температурах и относительно низком давлении германа (кривые $1, l^{\prime}$ на рис. $5, a$ ). В этом случае распад молекул $\mathrm{GeH}_{3}$ на чистой поверхности германия для разных механизмов десорбции водорода с поверхности характеризуется малой величиной энергии активации порядка $E_{a} \approx \pm 0.05 \mathrm{eV}(\sim 1 \mathrm{kkal} / \mathrm{mol})$. Повышение давления германа в установке UHVCVD (кривая 2) приводит к значительному (до $1 \mathrm{eV}$ ) повышению скорости поверхностного распада молекул гермила, указывая на возрастающую роль в процессе поверхностного пиролиза водорода, поступающего в объем реактора при распаде молекул гидрида. Сопоставление кривых на рис. $5, a, b$ показывает еще более сильный рост с температурой параметра $v_{\mathrm{GeH}_{3}}$ при переходе от роста пленок $\mathrm{Ge}$ в условиях сверхвысокого вакуума (кривые 1,2 ) к более низкому вакууму в установках проточного типа (кривые 3-5). Повышение концентрации водорода в реакторе установок, обусловленное ростом концентрации гидрида (а возможно и молекулярного водорода) в ростовой камере, приводит к бо́льшим значениям энергии активации процесса пиролиза. Вычисленные в области повышенных значений температур и низких давлений гидрида значения энергии активации поверхностного распада адсорбируемых молекул гермила (рис. 5,a) достаточно близки к энергии активации высокотемпературного распада молекул силила $\left(\mathrm{SiH}_{3}\right)$ на поверхности кремния (порядка $2-4 \mathrm{kkal} / \mathrm{mol}[6,34])$. Это указывает на близость каталитических свойств чистых $\mathrm{Si}-$ и $\mathrm{Ge}-$ поверхностей по отношению к адсорбируемым фрагментам молекул гидридов $\mathrm{Si}$ и $\mathrm{Ge}$ соответственно [36].

В области низких ростовых температур $\left(T_{g r}<400^{\circ} \mathrm{C}\right)$, когда распад адсорбируемых молекул гермила происходит на частично заполненной водородом поверхности германия, изменения кинетических параметров выражены сильнее. Уменьшение степени покрытия поверхности водородом, появляющееся, например, при смене механизма десорбции водорода (параметр $m=1$ меняется на $m=2$ ), понижение температуры приводит к заметному изменению величины и характера поведения параметра $v_{\mathrm{GeH}_{3}}$ (сплошные и штриховые линии на рис. 5 соответственно). Наблюдаемая при низкой температуре смена знака энергии активации процесса распада молекул гермила, вероятнее всего, аналогично тому, что имеет место для молекул силила на кремнии [37], связана с изменением роли механизмов, отвечающих за распад адсорбируемых молекул гидрида и протекающих без участия, либо с участием поверхностного водорода. В последнем случае часть атомов водорода с захваченной поверхностью германия молекулы гидрида $\mathrm{GeH}_{3}$ и атомы адсорбированного атомарного водорода могут связываться в молекулу водорода, обеспечивая его эффективную десорбцию с поверхности пластины. Разные механизмы перехода атома водорода с молекулы на поверхность, реализуемые с участием либо без участия в процессе поверхностного распада молекулы гидрида адсорбированных атомов водорода, характеризуются значительной разницей в энергетике высокотемпературного и низкотемпературного процессов пиролиза, обусловливая соответственно разницу энергий активации этих процессов в указанных интервалах температур. Смена механизма распада молекулы в диапазоне промежуточных температур роста $400-500^{\circ} \mathrm{C}$ и одновременное изменение степени покрытия поверхности атомами водорода является основной причиной наблюдаемого поведения температурной зависимости кинетических коэффициентов в области низких ростовых температур.

В последние годы при выращивании из гидридов пленок германия прослеживается явная тенденция к замене высоковакуумного ростового оборудования (UHVCVD) более производительными и менее дорогостоящими LPCVD-установками проточного типа. Для последних в области повышенных ростовых температур (рис. 1,a) 
наблюдается хорошо выраженная зависимость скорости роста слоев $\mathrm{Ge}$ от температуры, что не является характерным для высоковакуумных ростовых установок. Одновременно скорость роста пленок Ge в LPCVD-реакторе растет с повышением в нем давления газов (германа и молекулярного водорода). В связи с этим представляется важным понять, насколько сильно наблюдаемые отклонения в поведении скорости роста скажутся и на поведении изучаемых кинетических коэффициентов. Сопоставление кривых 1,2 на рис. 5, а с кривыми 3-5 на рис. $5, b$ показывает, что наиболее значительные различия в поведении скоростей поверхностного пиролиза молекул гермила наблюдаются в области повышенных $\sim(450-700)^{\circ} \mathrm{C}$ ростовых температур. В системах проточного типа, работающих при пониженном давлении газов, значения энергии активации процесса поверхностного пиролиза молекул гермила в рассматриваемом температурном интервале существенно возрастают (до величин $E_{a} \sim 4 \mathrm{eV}$ ).

$\mathrm{C}$ ростом давления германа в установке концентрация гермила на поверхности Ge практически не меняется (рис. 4). Однако величина давления германа в реакторе оказывает существенное влияние на скорость поверхностного пиролиза адсорбируемых поверхностью германия молекул гермила. С ростом давления $\mathrm{P}_{\mathrm{GeH} 4}$ на порядок величины скорость распада захватываемых молекул в обоих случаях (UHVCVD и LPCVD) также возрастает примерно на порядок величины (рис. 5,c). При заданном давлении германа эта величина растет: a) со сменой механизма пиролиза (замена параметра $n=3$ на $n=1)$ - ср. кривые 1,2 с кривыми $1^{\prime \prime}, 2^{\prime \prime}$ на рис. $5, a$ и кривые $4,4^{\prime}$ на рис. $5, b$; b) в области низких температур со сменой механизма десорбции водорода (замена $m=1$ на $m=2$ ) - ср. кривые $i, i^{\prime}$ на рис. $5, a, b$. В области повышенных (порядка $600^{\circ} \mathrm{C}$ ) температур (рис. 5,c) при заданном парциальном давлении германа скорость поверхностного пиролиза молекул гермила аналогично пиролизу молекул силила [22] падает с ростом давления молекулярного водорода в реакторе. Данный эффект можно объяснить влиянием на процесс пиролиза приповерхностного водорода. Концентрация последнего возрастает у поверхности с ростом давления $\mathrm{P}_{\mathrm{H} 2}$, приводя к изменению характеристик приповерхностного потенциала и оказывая влияние на каталитические свойства поверхности.

\section{Заключение}

В рамках кинетической модели, принимающей во внимание на поверхности германия только адсорбируемые молекулы гермила $\left(\mathrm{GeH}_{3}\right)$ и атомы водорода, проведены оценки величины и определен характер температурного поведения кинетических параметров, ответственных за скорости протекания на поверхности растущего слоя германия процессов десорбции водорода, захвата молекул германа и последующего их распада. В интервале температур $300-700^{\circ} \mathrm{C}$ для разных ростовых методов, реализуемых на базе высоковакуумных (UHVCVD) либо проточных при пониженном давлении (L(R)PCVD) установок, проанализировано влияние на скорость поверхностного пиролиза молекул гермила характера (ассоциативный либо диссоциативный) процессов десорбции водорода с поверхности. Определен интервал допустимых значений коэффициента захвата поверхностью германия молекул германа. Показано, что скорость распада на поверхности германия адсорбируемых молекул гермила в зависимости от температуры обнаруживает немонотонное поведение и линейно растет с ростом давления германа в реакторе. Скорость и характер температурной зависимости процесса пиролиза определяется также тем, на каком этапе процесса сорбции происходит захват поверхностью водорода с адсорбируемой молекулы гидрида. В методе UHVCVD энергия активации процесса распада захватываемых ростовой поверхностью молекул гермила в интервале повышенных температур и низких давлений принимает низкие значения, сравнимые с энергией активации процесса распада молекул силила $\left(\mathrm{SiH}_{3}\right)$ на кремнии. Величина энергии активации процесса пиролиза зависит от давления рабочих газов в реакторе и растет с повышением давления как германа, так и молекулярного водорода.

\section{Благодарности}

В заключении авторы выражают глубокую признательность д.ф.м.н. Орлову Л.К. за предоставление информации по скоростям роста слоев германия и оказанную помощь при написании работы.

\section{Финансирование работы}

Работа выполнялась в рамках и при финансовой поддержке Российского фонда фундаментальных исследований, проект 18-42-520062.

\section{Конфликт интересов}

Авторы заявляют, что у них нет конфликта интересов.

\section{Список литературы}

[1] J. Shi, E.S. Tok, H.C. Kang. J. Chem. Phys., 127, 164713 (2007). DOI: $10.1063 / 1.2799980$

[2] R.Q.M. Ng, E.S. Tok., H.C. Hang. J. Chem. Phys., 131, 044707 (2009). DOI: $10.1063 / 1.3191780$

[3] M. Shinohara, A. Seyama, Y. Kimura, M. Niwano. Phys. Rev. B, 65, 075319 (2002). DOI: 10.1103/PhysRevB.65.075319

[4] R.D. Smardon, G.P. Srivastava. J. Chem. Phys., 123, 174703 (2005). DOI: 10.1063/1.2087347

[5] N. Taylor, H. Kim, P. Desjardins, Y.L. Foo., J.E. Greene. Appl. Phys. Lett., 76, 2853 (2000).

DOI: $10.1063 / 1.126495$ 
[6] Л.К. Орлов, Н.Л. Ивина, Т.Н. Смыслова. ЖОХ, 83 (12), 1975 (2013). [L.K. Orlov, N.L. Ivina, T.N. Smyslova. Russ. J. General Chem., 83 (12), 2240 (2013). DOI: $10.1134 / \mathrm{S} 1070363213120037]$

[7] J.M. Hartmann, V. Mazzocchi, F. Pierre, J.P. Barnes. ECS Transactions., 86, 219 (2018).

[8] H. Kim, J.E. Greene. Surf. Sci., 504, 108 (2002).

[9] T.I. Kammins, E.C. Carr, R.S. Williams, S.J. Rosner. J. Appl. Phys., 81, 211 (1997). DOI: 10.1063/1.364084

[10] B. Cunningham, J.O. Chu, S. Akbar. Appl. Phys. Lett., 59, 3574 (1991). DOI: 10.1063/1.105636

[11] M. Halbwax, D. Bouchier, V. Yam, D. Débarre, L.H. Nguyen, Y. Zheng, P. Rosner, M. Benamara, H.P. Strunk, C. Clerc. J. Appl. Phys., 97, 064907 (2005). DOI: 10.1063/1.1854723

[12] J.M. Hartmann, F. Bertin, G. Rolland, M.N. Semeria, G. Bremond. Thin Solid Films., 479, 113 (2005). DOI:10.1016/j.tsf.2004.11.204

[13] C. Li, S. John, E.S. Quinones. J. Vac. Sci. Technol., A14, 170 (1996). DOI: $10.1116 / 1.579915$

[14] K.J. Kim, M. Suemitsu, M. Yamanaka, N. Miyamoto. Appl. Phys. Lett., 62, 3461(1993). DOI: 10.1063/1.109049

[15] Л.К. Орлов, С.В. Ивин. Химическая физика, 35 (3), 36 (2016). DOI: 10.7868/S0207401X16030055

[L.K. Orlov, S.V. Ivin. Russ. J. Phys. Chem. B, 10 (2), 219 (2016). DOI: 10.1134/S1990793116020056]

[16] Л.К. Орлов, С.В. Ивин. ЖОХ, 85 (12), 1951 (2015). [L.K. Orlov, S.V. Ivin. Russ. J. General Chem., 85 (12), 2686 (2015). DOI: 10.1134/S107036321512004X]

[17] J.M. Hartmann, A. Abbadie, A.M. Papon, P. Holliger, G. Rolland, T. Billon, J.M. Fédéli, M. Rouviére, L. Vivien, S. Laval. J. Appl. Phys., 95, 5905 (2004). DOI: $10.1063 / 1.1699524$

[18] Л.К. Орлов, В.А. Толомасов, А.В. Потапов, В.И. Вдовин. Изв. вузов. Материалы электронной техники, 2, 30 (1998).

[19] L.K. Orlov, V.A. Tolomasov, A.V. Potapov, Yu.N. Drozdov., V.I. Vdovin. IEEE Trans. Semicond. Manufacturing, V. SIMC-9. 215 (1996).

[20] P.M. Garone, J.C. Sturm, P.W. Schwartz. Appl. Phys. Lett., 56, 1275 (1990). DOI:10.1063/1.102535

[21] S. Kobayashi, N. Mikoshiba, T. Matsuura, M. Sakuraba, J. Murota. J. Cryst. Growth., 174, 686 (1997).

[22] T.N. Adam. In the book: SiGe and Si Strained-Layer Epitaxy for Silicon Heterostructure Devices / ed. J.D. Gressler. (CRC Press, Tailor \& Francis Groop, London, NY., 2008), p. 7.

[23] J. Aubin, J.M. Hartmann, V. Benevent. Thin Solid Films, 602, 36 (2016). DOI:10.1016/j.tsf.2015.07.024

[24] J. Aubin, J.M. Hartmann, M. Bauer, S. Moffatt. J. Cryst. Growth., 445, 65 (2016). DOI: $10.1016 /$ j.jcrysgro.2016.04.018

[25] F. Leys, R. Bonzom, R. Loo, O. Richard., B. De Jaeger., J. Van Steenbergen, K. Dessein, T. Conard, J. Rip., H. Bender, W. Vandervorst, M. Meuris, M. Caymax. Thin Solid Films, 508, 292 (2006). DOI:10.1016/j.tsf.2005.08.411

[26] T. Bramblett, Q. Lu, N. Lee, N. Taylor, M. Hasan, J.E. Green. J. Appl. Phys., 77, 1504 (1995). DOI: 10.1063/1.358901

[27] Л.К. Орлов, Т.Н. Смыслова. ФТП, 39 (11), 1320 (2005). [L.K. Orlov, T.N. Smyslova. Semiconductors, 39 (11), 1275 (2005).]

[28] L. Surnev, M. Tikhov. Surf. Sci., 138, 40 (1984).

[29] L.B. Lewis., J. Segall, K.C. Janda. J. Chem. Phys., 102, 7222 (1995). DOI: $10.1063 / 1.469117$
[30] E.S. Tok, S.W. Ong, H.Ch. Kang. J. Chem. Phys., 120, 5424 (2004). DOI: 10.1063/1.1645510

[31] B.A. Ferguson, C.T. Reeves, D.J. Safarik, C.B. Mullins. J. Phys. Chem., 113, 2470 (2000).

[32] J.S. Lin, L.F. Lee. Int. J. Quantum Chem., 97, 736 (2004).

[33] J.S. Lin, Y.T. Kuo. Thin Solid Films, 370, 192 (2000).

[34] J.K. Kang, C.B. Musgrave. Phys. Rev. B, 64, 245330 (2001). DOI: 10.1103/PhysRevB.64.245330

[35] A.R. Brown, D.J. Doren. J. Chem. Phys., 110, 2643 (1999). DOI: $10.1063 / 1.477986$

[36] Л.К. Орлов, Н.Л. Ивина, В.А. Боженкин. ФТП, 53 (7), 995 (2019). DOI: 10.21883/JTF.2021.06.50875.82-20 [L.K. Orlov, N.L. Ivina, V.A. Bozhenkin. Semiconductors, 53 (7), 979 (2019). DOI: 10.1134/S1063782619070182]

[37] Л.К. Орлов, Т.Н. Смыслова. ЖТФ. 82 (11), 83 (2012). [L.K. Orlov, T.N. Smyslova. Tech. Phys., 57 (11), 1547 (2012). DOI: $10.1134 / \mathrm{S} 1063784212110187]$ 